\title{
TRAJETÓRIAS DE USO DAS TECNOLOGIAS DE COMUNICAÇÃO: AS FORMAS DE APROPRIAÇÃO DA CULTURA DIGITAL COMO DESAFIOS DE UMA 'SOCIEDADE DO CONHECIMENTO' *
}

\section{PATHS OF USES OF COMMUNICATION TECHNOLOGIES: THE FORMS OF APPROPRIATION OF DIGITAL CULTURE AS A CHALLENGE FOR A 'SOCIETY OF KNOWLEDGE'}

\author{
SERGE PROULX ${ }^{* *}$
}

\begin{abstract}
RESUMO: Com a notória expansão da mediação de práticas comunicativas em redes digitais, aconteceram mudanças significativas no campo sociocultural. Estamos apenas começando a perceber as consequências dessas mudanças e elas já levantam várias questões. Onde tais aparatos técnicos aparecem em práticas de sociabilidade e solidariedade, e na própria natureza do laço social? Que desafios econômicos, políticos e éticos estão relacionados a esses novos modos de intercâmbio, coordenação e comunicação? Nossa resposta está estruturada em torno de duas questões transversais colocadas em sentido oposto ao das trajetórias de uso da rede. Em primeiro lugar, o fato de se ter uma participação em larga escala numa "sociedade de conhecimento" requer apropriação de um núcleo de conhecimentos técnicos relacionados? E, em segundo lugar, deveríamos esperar a emergência de uma nova forma de pensamento, baseada na cooperação, troca e dom, associada ao uso intensivo de redes digitais de comunicação?

Palavras-chave: comunicação de massa; comunicação humana; tecnologia da informação; entorno sociocultural; rede de telecomunicação; cultura; técnica e sociedade.
\end{abstract}

\begin{abstract}
With the marked expansion of mediation of communicative practices in digital networks, signi?cant changes have happened in the sociocultural landscape. We are only just beginning to grasp the consequences of these changes, and they raise a number of issues. Where do such socio-technical apparatuses ?gure in the practices of sociability and solidarity, and indeed in the very nature of the social bond? What economic, political, and ethical challenges are linked to these new modes of exchange, coordination, and communication? Our response is structured through two cross-cutting questions, situated upstream from trajectories of network use. First, does the fact of large-scale participation in a «knowledge-based society» require appropriation of a core set of related technical knowledges? And, second, should we expect the emergence of a new form of thinking based on cooperation, exchange, and gift-giving, and associated with intensive use of digital communication networks?
\end{abstract}

Keywords: mass communication; human communication; information technology; sociocultural environment; telecommunication network; culture; technique, society.

* Tradução da comunicação "Trajectoires d'usages des Technologies de communication: les formes d'appropriation d'une culture numérique comme enjeu d'une 'société du savoir' “, publicada em Annales des télécommunications. 57, n 3-4, 2002, pp. 180-189. As organizadoras deste número agradecem ao autor do texto pela mediação no processo de autorização para tradução.

** Professor do Département des communications, Université du Québec à Montréal, Montreal (Canadá) 
A expansão das práticas de comunicação mediadas por redes digitais traz mudanças significativas no contexto sociocultural e as conseqüências dessas mudanças começam apenas a serem percebidas. Questões surgem, e são muitas: qual a incidência desses dispositivos sociotécnicos nas práticas de sociabilidade e de solidariedade, até mesmo na própria natureza das relações sociais? Quais são os desafios culturais e éticos relacionados a essas novas práticas de interação, coordenação e comunicação? Tentaremos neste trabalho trazer elementos para responder a uma questão mais específica, que se coloca em função e além da percepção das trajetórias de uso das tecnologias de comunicação e que diz respeito ao desenvolvimento em grande escala das práticas de comunicação mediadas por redes digitais. Questão que formularemos da seguinte forma: a participação ativa de um maior número de indivíduos numa 'sociedade baseada no conhecimento' torna necessária a apropriação de um núcleo mínimo de saberes técnicos associados a essa nova cultura, dita digital? O uso das expressões 'sociedade baseada no conhecimento', ou 'sociedade do conhecimento' aponta para um traço caracterizador das transformações estruturais que atravessam nossas sociedades contemporâneas: a produção e a difusão da informação e do conhecimento constituem hoje os motores da mudança econômica e social.

\section{TRAJETÓRIAS DE USO}

Interessa ao nosso grupo de pesquisa atualmente traçar uma cartografia das trajetórias de uso das tecnologias de informação e comunicação (TIC) através da observação das práticas e de entrevistas com usuários, ou seja, interessa-nos a identificação, numa perspectiva diacrônica, das modificações ocorridas nos percursos de uso das TIC- e sobretudo hoje, de uso da Internet - por grupos específicos de usuários (mudanças no plano das histórias pessoais dos sujeitos, suscitadas por uso iterativo ou recorrência de práticas originalmente desenvolvidas com antigos dispositivos; emergência de novos hábitos de uso da mídia; reorientação de práticas antigas) ${ }^{1}$. Essas transformações se mesclam por um lado com as ações situadas dos indivíduos e dos grupos em seus respectivos contextos de vida privada (família, consumo, etc), de vida profissional e de vida pública (ou cidadã). Por outro lado, se inscrevem em continuidade e em relação complementar com o ordinário ou comum da comunicação (cf. os trabalhos de CERTEAU) preexistente (por exemplo: padrões de conversa face a face; hábitos de uso do telefone, do computador, da copiadora, da televisão, do rádio etc.).

E a explosão do número de novos artefatos digitais de comunicação postos em funcionamento nos últimos anos, bem como a proliferação de aplicativos computacionais e de suas possibilidades de uso é um fenômeno que nos obriga a adotar uma perspectiva mais abrangente para evitar o risco de uma análise muito fragmentada. Desse modo, um estudo apropriado dos usos da Internet deve considerar o fato de que a rede é apenas um

${ }^{1}$ 1. A primeira seção apresenta contribuições de Guillaume Latzko-Toth, assistente do Grupo de pesquisa sobre as mídias (GRM). Agradeço-lhe calorosamente por ter aceitado o compartilhamento das idéias que desenvolvemos em sinergia e das quais ele é co-autor. Agradeço também aos seguintes membros do GRM que comentaram textos preliminares a esta comunicação: Micheline Frenette, Jacques Lajoie, Florence Millerand, Johanne Saint-Charles, Michael Totschnig. 
componente infraestrutural de uma enorme constelação de artefatos técnicos empresariais, domiciliares ou nômades, que vão desde os telefones celulares aos apoios digitais personalizados, passando pelo fax, pelos computadores portáteis ou de mesa, pelos leitores de áudio com ou sem dispositivo de gravação, pelos leitores de DVD, pelos livros eletrônicos, pelos televisores, pelas antenas parabólicas, pelos terminais de jogos e pelos aparelhos de TV digital. Deve considerar também a utilização de outras tecnologias de rede, além da Internet. Na verdade, embora o termo 'Internet' ainda seja útil como designação genérica desse conjunto reticular de dispositivos técnicos, as práticas de comunicação mediadas pelas redes digitais abrangem mais que a rede Internet, sendo que essa é uma rede cada vez mais orientada por uma lógica comercial.

O estudo ampliado das trajetórias de uso deve possibilitar a compreensão de porque um único aplicativo Internet, dentre vários concorrentes, acaba muitas vezes se impondo para um determinado grupo social e para um determinado contexto de utilização. E em que medida se poderia estabelecer correlações entre a preferência por um aplicativo e a utilização anterior ou paralela, quando não simultânea, de certos dispositivos? (JONES, 1999). Nesse sentido é que nos propomos a estudar as trajetórias individuais e coletivas de uso, isto é, os percursos de indivíduos e de grupos (usos sucessivos e concomitantes) através de constelações de objetos de comunicação antigos, contemporâneos, emergentes ou em declínio. $\mathrm{O}$ estudo das transformações das redes sociais nas quais se inserem os usuários deve possibilitar a identificação das formas que assumem os coletivos que emergem com a escolha e a estabilização das práticas de comunicação via Internet (WELLMAN, 1999). E essa é uma maneira de se contemplar a dimensão coletiva das práticas de comunicação mediadas pelas redes digitais.

Mas além da proliferação dos artefatos, o que se vê é o surgimento de um número cada vez maior de possibilidades de comunicação entre eles, quando não de fusão pura e simples (HERMAN \& SWISS, 2000). Ora, essa fluidez cada vez maior nos leva a realçar as continuidades que existem entre diferentes usos que podem ser identificados como equivalentes funcionais de uma mesma prática de comunicação, sem que seja necessário o atrelamento a uma determinada modalidade técnica. Assim, o objetivo não é o de propor uma tipologia exaustiva e conclusiva das práticas de comunicação focalizadas, e sim o de fazer ver regularidades onde um primeiro olhar só vê uma justaposição de objetos e de usos disparatados, o de iluminar os processos pelos quais certos usos tendem a se manter, a se estabilizar, até a migrar de um dispositivo para outro, ao passo que outros usos previstos pela indústria são abandonados num período mais ou menos curto. Esse estudo adquire maior interesse hoje, na medida em que a diversidade da oferta de tecnologias é obra de uma diversidade ainda maior de atores sociais (MACKENZIE \& WAJCMAN, 1999).

Retomando o que foi dito no início, a expansão considerável das práticas de comunicação mediadas por redes digitais traz mudanças significativas no contexto sociocultural com conseqüências ainda pouco conhecidas. E as questões que surgem são muitas: qual a incidência desses dispositivos sociotécnicos em nossas práticas de sociabilidade e de solidariedade, até mesmo na própria natureza das relações sociais? Quais são os desafios econômicos, políticos e éticos relacionados a essas novas práticas de interação, de coordenação e de comunicação? Mas o momento não é mais o dos discursos proféticos - eufóricos ou catastróficos - sobre os 'impactos' da Internet em nossas sociedades. A observação empírica contradiz constantemente várias dessas predições que 
poderíamos qualificar de unidimensionais para realçar a complexidade dos processos relacionados à concepção, à valorização, à apropriação e à integração desses dispositivos de comunicação nos diferentes contextos das práticas cotidianas.

\section{PRIMEIRAS CONTROVÉRSIAS A RESPEITO DA CULTURA TÉCNICA}

Os fundamentos de uma 'sociedade baseada no conhecimento' deveriam estar na apropriação, pela maioria de seus membros, de uma cultura técnica específica, a digital - a mesma cultura que está na base da construção das redes? Se a resposta for sim, a elucidação das condições precisas para uma apropriação individual e coletiva dessa cultura torna-se um fator primordial para a organização da vida em sociedade (FRENETTE, 1997; HARD \& JAMISON, 1998). Por apropriação, estamos compreendendo a integração criativa de elementos significativos dessa cultura na vida cotidiana dos usuários e das comunidades (PROULX, 1988). A apropriação construtiva dessa cultura aparece, assim, como um componente chave da inserção dos indivíduos e das comunidades na 'sociedade do conhecimento'.

Essa questão está historicamente relacionada a um conjunto de controvérsias divulgadas no início da década de 1980 para a opinião pública francesa e suscitadas sobretudo pelo Centro de pesquisas sobre a cultura técnica (CRCT) e por universitários e pesquisadores na época envolvidos no desenvolvimento de um programa de pesquisa e ensino sobre 'Ciências, técnicas e sociedade' (STS). Os intelectuais franceses se reuniam em torno de um projeto de superação necessária do que havia sido apontado pelo intelectual britânico Charles P. Snow numa conferência proferida em 1959, quando chamou a atenção para o fosso que separava a cultura literária tradicional e a cultura científica (SNOW, 1968). Mas enquanto os debates da opinião pública britânica se deram sobretudo durante a década de 1960 - Snow publicou, inclusive, novas reflexões em 1963 - e se desenvolveram em função da probabilidade, anunciada por Snow, do surgimento de uma 'terceira cultura' (no caso, a cultura das ciências humanas), os intelectuais franceses dos anos 1980 defendiam a necessidade de se divulgar e de se dar acesso à cultura das técnicas, instrumento essencial, segundo eles, para que as pessoas tivessem orientação e controle frente às formidáveis transformações de ordem técnica que estavam ocorrendo. Desse modo, Jocelyn de Noblet, organizador do CRCT e responsável pela publicação da revista Cultura Técnica, coordenou em 1981 a publicação de um Manifesto para o desenvolvimento da cultura técnica, prefaciado por André Leroi-Gourhan (CRCT, 1981). Nesse manifesto lê-se o seguinte diagnóstico:

«Parece, portanto, evidente que aquele que não tem cultura técnica vive na ignorância de seu próprio meio. Encontra-se, pela mesma razão, duplamente alienado: por um lado não domina o seu meio e, por outro, essa falta de domínio o coloca socialmente na dependência permanente de organizações e indivíduos que têm as competências que ele não tem. (...) Acreditamos, portanto, que uma cultura técnica é necessária porque pode ser compreendida como a possessão de um mínimo de conhecimentos e de habilidades que nos permitam a reapropriação do meio em que vivemos. Queremos dizer que ela é uma das condições a partir das quais é possível se apropriar das técnicas e que a partir dela se pode mais facilmente evitar a dependência, até mesmo a alienação» (CRCT, 1981, p. 11-12) 
Essa maneira de colocar a questão cristalizou-se na França no momento em que a microinformática irrompeu na cena pública. Nos anos 1980, não era raro alguém discorrendo sobre a necessidade de se assegurar para todos e para cada um as condições sociais para uma 'alfabetização informática', expressão utilizada para designar o mínimo necessário e suficiente em termos de conhecimentos e de habilidades a serem adquiridos, de modo a que um maior número de indivíduos pudessem se apropriar da cultura informática (PROULX, 1990). Tais debates duraram um certo tempo, talvez por causa do caráter fundamentalmente utópico do projeto, talvez também porque essa primeira formulação da questão de uma 'cultura técnica' não conseguia traduzir adequadamente a finalidade e a materialidade das práticas concretas de apropriação da microinformática. Mas eis que essa questão ressurge nas discussões contemporâneas sobre apropriação da Internet e sobre o desenvolvimento de uma cultura dita digital, ou seja, baseada no domínio das tecnologias digitais de informação e comunicação.

\section{POR UMA REFORMULAÇÃO DA QUESTÃO DA CULTURA TÉCNICA: ENTRE O ARTEFATO COGNITIVO E O SOFTWARE LIVRE}

Um dos limites da questão da cultura técnica, tal como foi formulada na década de 1980, é a de postular implicitamente que o suporte da inteligência técnica necessária à apropriação das máquinas de comunicação deveria ser necessariamente e exclusivamente o indivíduo humano. Ora, os trabalhos dos últimos vinte e cinco anos sobre as interações humano-computador e sobre o design de interfaces mostraram que os próprios computadores poderiam se constituir como um suporte da inteligência técnica. A noção de artefato cognitivo apresentada por Donald Norman evidencia o fato de que o objeto técnico pode ser considerado como um recurso cognitivo, como um suporte estratégico na realização de atividades cognitivas pelo indivíduo humano que utiliza o objeto técnico (NORMAN, 1993):

\footnotetext{
«A ideia mestra nesse caso consiste em considerar os objetos técnicos como artefatos que agem como parceiros na atividade cognitiva daquele ou daquela que o utiliza. Eles podem, assim, ser considerados como recursos que permitem facilitar as tarefas cognitivas de atenção, raciocínio, memorização, planejamento, etc, na medida em que assumem uma parte da atividade cognitiva do usuário.» (MILLERAND, 2001, p. 3-4)
}

Tentemos, então, tirar as conseqüências de uma abordagem como essa para repensarmos a questão da cultura técnica. Em primeiro lugar, não se pode negar o fato de que a aprendizagem de um mínimo de habilidades técnicas é necessária ao agente humano para que ele possa controlar adequadamente o dispositivo de comunicação. Mas, ao mesmo tempo, com o desenvolvimento progressivo de sistemas e interfaces cada vez mais 'inteligentes' (no sentido informático do termo), conhecer o processo de apropriação humana do objeto técnico implica em conhecer também a 'inteligência técnica' que já está embutida na máquina (para uma reflexão pertinente sobre o caso dos agentes inteligentes em interfaces, ver ERICKSON, 1997). Interfaces amigáveis, sistemas mais centrados no usuário, automatização de operações iniciadas pelo agente humano, são componentes introduzidos progressivamente no design dos computadores e dos dispositivos digitais. Desse modo, 
enquanto no início dos anos 1980 se podia supor que uma apropriação adequada da microinformática exigiria o domínio de um mínimo de linguagem de programação - o que na época representava o mais alto nível de apropriação da parte de um não profissional da informática - pode-se supor hoje que um usuário que domina perfeitamente o meio digital não necessitaria aprender programação. Pelo menos, essa seria uma posição possível nos debates sobre identificação de competências mínimas necessárias para circular com facilidade no universo digital.

Dessa forma, uma parte da inteligência técnica necessária ao auto-controle e à não alienação do agente humano já está embutida nos dispositivos interativos que se oferecem a ele. A apropriação da tecnologia fica, pois, facilitada. O que não significa, porém, que o domínio do ambiente digital não seja por si mesmo problemático: a aprendizagem do modo operatório do artefato cognitivo é, na verdade, uma etapa muito importante do processo de apropriação (etapa em que se formam e se transformam as representações mentais dos objetos técnicos, produzidas pelos agentes) $)^{2}$. Para melhor compreender essa evolução da arquitetura dos objetos técnicos, se poderia fazer um paralelo com o automóvel: o domínio da direção de um carro é hoje completamente independente do conhecimento que se possa ter da mecânica automotiva.

A evolução dos objetos técnicos exige, portanto, uma reavaliação da natureza da cultura técnica hoje necessária para uma apropriação adequada dos dispositivos de comunicação mediada pelas redes digitais. Uma parte inicial dessa cultura técnica consiste na sensibilização geral (filosófica, sociológica) do lugar da técnica na sociedade contemporânea, das relações complexas de co-determinação que unem tecnologias e sociedades. Eé através de um trabalho prático de sensibilização e de autosensibilização (de natureza psicosociológica) para as atitudes (muitas vezes inconscientes) e para as emoções dos agentes humanos frente o fenômeno técnico que se tornará possível a apropriação dessa primeira parte de conhecimentos. A segunda parte diz respeito ao domínio mínimo dos ambientes e códigos digitais, e das interfaces necessárias à 'navegação' no universo computacional. A terceira parte é a da aprendizagem dos modos de comunicação próprios a esse novo ambiente (práticas interacionais, uso de códigos éticos aplicáveis à gestão das relações interacionais on-line, novos modos cooperativos de cumprimento de tarefas envolvendo interlocutores conectados em redes humanas).

Mas esse movimento de introdução de automatismos e de interfaces inteligentes no design dos dispositivos de comunicação representa apenas um dos pólos do processo dinâmico de reformulação necessária da questão da cultura técnica. O outro polo é representado pelos porta-vozes do 'movimento do software livre'. Trata-se de um movimento em reação à difusão maciça dos 'sistemas proprietários', os quais obrigam os usuários a circularem no interior de um universo digital fechado, em que as possibilidades de mudança de estrutura ou de código são extremamente limitadas. ${ }^{3}$ Os participantes desse movimento

${ }^{2}$ Contribuições trazidas por Florence Millerand.

3 3. Nossas considerações sobre a informática livre são inspiradas num projeto de pesquisa em curso, juntamente com Thierry Bardini, professor da Universidade de Montreal, e a participação de Michael Totschnig, doutorando da UQAM (Projeto «La liberté dans un univers de règles. Analyse socio-historique du mouvement informatique libre» (A liberdade num universo de regras. Análise sociohistórica do movemento informática livre), subvencionado pelo CRSH (2001-2004). 
defendem o acesso livre aos códigos-fonte dos programas para toda pessoa que queira reutilizá-los ou participar de seu desenvolvimento. Mesmo se o movimento 'software livre' deve muito de seu reconhecimento público ao sucesso do sistema Linux, seu significado cultural e social vai muito além disso. Enquanto novo modo de produção e de distribuição de conhecimentos, o movimento do software livre mostra claramente o poder criativo das redes de colaboradores, reaproximando usuários e criadores, estabelecendo pontes cruciais entre diferentes domínios do conhecimento. Ele representa, na verdade, um processo de design que é tanto técnico quanto social, um conjunto de sistemas informáticos e organizacionais que permitem a coordenação das contribuições de um conjunto significativo de indivíduos - às vezes dispersos tanto cultural quanto fisicamente - com vistas à construção coletiva de um bem público. Os valores essenciais desse movimento começam a difundir-se além das fronteiras disciplinares, sociais ou culturais. Comunidades 'software livre' se constituem em inúmeros países e se comunicam através de listas de discussão e de ações cooperativas no campo do desenvolvimento de sistemas computacionais.

As ações desse movimento se inscrevem no que Bernard Conein et Nicolas Auray designam por 'politizaçào dos usos' ${ }^{4}$, isto é, uma orientação do olhar do observador para os modos como o uso da informática pode contribuir para a constituição de uma "vida em comum', e de que modo o uso de objetos técnicos pode favorecer um melhor controle, por parte de cada indivíduo, sobre sua própria vida. Uma contradição interessante surgiu com o projeto de estender a informática livre ao 'grande público'. Tendo sido até então um movimento de usuários que também eram da área (criadores), que formas poderiam adquirir os produtos do software livre se os organizadores do movimento se propusessem a entrar no mercado massificado? Que nível de aquisição de cultura técnica seria necessário para que um usuário comum pudesse participar plenamente do movimento? Os responsáveis pelo sistema livre desejam que os usuários comuns se apropriem de um mínimo de conhecimentos de informática para usufruírem realmente dos benefícios do movimento. Mas é aí que dificuldades relativas a objetivos em princípio incompatíveis podem surgir: os objetivos do movimento software livre, constituído de especialistas em informática, versus as motivações dos usuários comuns. Parece evidente, por exemplo, que a maioria dos usuários comuns não deseja criar novos sistemas nem adquirir de forma obrigatória conhecimentos técnicos que se mostram dispensáveis quando utilizam sistemas inteligentes e competentes.

Mas por outro lado, essa incompatibilidade parece querer se deslocar ${ }^{5}$. Se é verdade que o software livre nasceu das necessidades de certos especialistas desejosos de obterem ferramentas versáteis e competentes (com possibilidade de construírem funcionalidades adaptadas a usos específicos) - esse desejo se contrapondo à tendência do mercado em fornecer ao grande público ambientes amigáveis mas com funcionamento pré-estabelecido - a tendência hoje é a dos cruzamentos e hibridizações entre as duas perspectivas. Nada impede hoje um artefato cognitivo de oferecer uma interface programável pelo usuário

${ }^{4}$ 4. Bernard Conein, Nicolas Auray, assim como Laurence Allard et Jean-Michel Cornu, trabalham atualmente no projeto «Internet comme technologie sociale: collectif par projet et politisation des usages» (Internet como tecnologia social: coletivo projetado e politização dos usos), subvencionado pelo programa RNRT (França).

${ }^{5}$ Commentário trazido por Michael Totschnig. 
(macro-comandos do sistema Word, programação do ambiente MacOS com Applescript, por exemplo). Estamos, então, diante de uma interface que supera as capacidades até aqui reservadas às interfaces de manipulação direta. As interfaces programáveis permitem, efetivamente, a realização de operações recorrentes e de tarefas mais complexas. Da mesma forma, encontramos atualmente no contexto do software livre, interfaces que possuem um primeiro nível de funcionamento amigável voltado para o usuário não experiente (KDE, Gnome, por exemplo), e que se sobrepõem a outros níveis mais avançados em que se faz necessário o domínio da programação pelo usuário. Se há uma oposição que permite que se contraste hoje a postura do software livre em relação à do mercado comercial, seria talvez a da questão da 'transparência' sugerida por Lessig (1999): as inteligências técnicas inscritas nos artefatos cognitivos podem ser objeto de uma crítica consistente da parte de seus usuários, ou de suas comunidades de usuários? Onde se coloca a responsabilidade ética dos criadores em relação aos artefatos que criaram?

\section{AEMERGÊNCIADE UMPENSAMENTO-REDE}

Estaríamos diante da emergência de uma nova forma de pensamento associada à utilização intensiva das redes de comunicação e que poderíamos chamar de pensamentorede? Tal forma de pensamento demandaria versatilidade, criatividade e flexibilidade na busca de soluções para os problemas. Essa racionalidade nova - orientada para a sinergia, para a inteligência coletiva e a cooperação - poderia gerar práticas sociais específicas e contribuir para o desenvolvimento de novas solidariedades num contexto social em que a competição entre indivíduos é altamente valorizada? Por outro lado, e contraditoriamente, esse movimento de valorização do pensamento em rede e do trabalho de cooperação em rede, de novas práticas de gratuidade, não seria apenas uma ilusão ideológica mascarando o jogo de lógicas sociais que operam num outro registro de relações de força ainda mais estruturantes?

A nova cultura digital que sucederia à cultura do impresso estaria relacionada a hábitos sociocognitivos específicos, após a razão gráfica (GOODY, 1979) e a razão computacional (BACHIMONT, 1999), que justificasse a ideia de um pensamento-rede? Tal termo remete não só aos fenômenos de inteligência distribuída, mas também às práticas individuais e organizacionais que mobilizam sistematicamente redes de atores nas ações cotidianas (CALLON et ali 1999). Mas além dos discursos entusiastas que fazem da rede um novo paradigma sociológico - ver, a título de contraste, a crítica de Boltanski \& Chiapello, 1999 sobre o 'novo espírito do capitalismo': o funcionamento em rede seria a nova modalidade de exploração capitalista - queremos avaliar em que medida se pode estabelecer relação entre as práticas individuais e coletivas de uso da Internet e o desenvolvimento de novas formas de sociabilidade (no seio das redes de indivíduos e de grupos). Veríamos emergir novos códigos de conduta mais voltados para a ajuda mútua, o dom, a cooperação e o apoio social, e isso em esferas diversificadas de atividade, ou mesmo em novas esferas? (GODBOUT, 1992). No caso afirmativo, isso indicaria que as práticas interativas relacionadas às redes sociotécnicas transversais - em relação complementar ou até algumas vezes em oposição às redes sociais tradicionais intrafamiliares ou intraorganizacionais - poderiam 
corresponder a uma nova dimensão das práticas de cooperação, confirmando assim a emergência de uma nova forma de pensamento (temática desenvolvida em outro lugar: PROULX, 2001).

\section{CONCLUSÃO: OS PERCURSOS DE USO SE FORMAM NUM CONTEXTO NORMATIVO EMTRANSFORMAÇÃO}

O último aspecto de nosso programa de pesquisa consiste em situar as descrições de trajetórias de uso em relação a um quadro de análise orientado para a compreensão das transformações do ambiente normativo que caracteriza as sociedades ocidentais contemporâneas. A globalização e a extensão das práticas de comunicação mediadas pelas redes digitais intensificam tensões que suscitam medos e esperanças ao mesmo tempo. Se, por um lado, as práticas novas são portadoras de fatores positivos de mudança, por outro, também fazem surgir novas formas de abuso e de intolerância. Daí o surgimento de debates sobre a questão de um novo ambiente normativo que estaria se constituindo na dinâmica de desenvolvimento das redes mundiais de comunicação ${ }^{6}$. Mas, diferentemente da lei, a norma (social ou técnica) não se faz por decreto: ela se constitui progressivamente através das práticas interindividuais e se torna a convenção que orientará as práticas futuras até que apareçam novas normas para substituir as antigas. Desse modo, o círculo dos criadores de normas sociais é sempre maior que o dos legisladores. $\mathrm{O}$ fato de se considerar, à maneira de Lessig, quatro tipos de normas - jurídicas e comerciais, mas também técnicas e sociais (LESSIG, 1999) - torna a abordagem produtiva para se escapar ao dilema em que nos colocamos sempre frente à questão: 'pode-se legislar sobre a Internet?' (KAHIN \& KELLER, 1997 ; LOADER, 1997). O legislador é, de fato, apenas um dos atores - num papel seguramente privilegiado - desse novo contexto normativo (LELONG \& MALLARD, 2000). Os demais atores agem em função do jogo do mercado econômico (jogo das corporações) ou na condição de porta-vozes da sociedade civil e, particularmente, dos movimentos sociais que a atravessam (CASTELLS, 1999). Mas a norma não é só sinônimo de coerção: ao evoluir, ela traz por sua vez transformações nos modos de se fazer e agir, superando a inércia das práticas sociais anteriores. O contexto normativo é, assim, atravessado pelas novas formas de expressão e de reivindicação relacionadas ou não ao uso de dispositivos interativos de comunicação. Os percursos de uso das tecnologias de informação e comunicação (TIC) heterogêneos, plurais e multifuncionais - se desenvolvem, portanto, num contexto normativo feito de regras, de procedimentos e de protocolos. Simultaneamente, tais trajetórias de uso, próprias de usuários comuns ou inovadores, podem romper em alguns momentos os quadros normativos coercitivos fazendo emergir novas práticas e novos usos significativamente diferentes dos de antes. Desse modo, novas regras e novos códigos de

${ }^{6}$ 6. Essas obervações estão relacionadas a um projeto de cooperação franco-quebequense, coordenado por Serge Proulx, Bernard Conein et Françoise Massit-Folléa, e envolvendo a participação de uma quinzena de pesquisadores. O título do projeto é «Coopération en réseau via Internet: création et diffusion de nouvelles normes dans un contexte de mondialisation», ?nanciado pelo Ministério das Relações Exteriores do governo francês e pelo Ministério das Relações Internacionais do governo do Québec (2000-2002). 
conduta substituem de forma dinâmica as antigas normas. Sob essa perspectiva, as trajetórias de uso não são apenas a tradução dos padrões habituais dos usuários: elas constituem ao mesmo tempo espaços fecundos de criatividade para a ação humana.

\section{REFERÊNCIAS BIBLIOGRÁFICAS}

BACHIMONT, B. (1999). L'intelligence arti?cielle comme écriture dynamique : de la raison graphique à la raison computationnelle, in J. Petitot, (éd.), Au nom du sens. Paris: Grasset.

BOLTANSKI, L.; CHIAPELLO, E. (1999). Le nouvel esprit du capitalisme. Gallimard: Paris.

CALLON, M. et ali., (1999). Réseau et coordination. Paris: Economica.

CASTELLS, M. , (1999). Le pouvoir de l'identité. Paris: Fayard.

CRCT, (1981). Manifeste pour le développement de la culture technique, Neuilly-sur-Seine. Centre de Recherche sur la Culture Technique.

ERICKSON, T. (1997). Designing Agents as if People Mattered, in BRADSHAW J.M., ed, Software Agents, Cambridge: MIT Press, p. 79-96, (1997).

FRENETTE, M. (1997). Interactive technology in the classroom: A case study with illiterate adults, in Kubey R., ed., Media Literacy in the Information Age, New Brunswick: Transaction, 377-402.

GODBOUT, J.T. (1992). L'esprit du don. Paris: La Découverte.

GOODY, J. (1979). La raison graphique. Paris: Minuit.

HARD, M.;JAMISON, A. (1998). The Intellectual Appropriation of Technology. Cambridge: MIT Press.

HERMAN, A.; SWISS, T. eds. (2000). The World Wide Web and Contemporary Cultural Theory. New York: Routledge.

JONES, S. (1999). Doing Internet Research. Critical Issues and Methods for Examining the Net. Thousand Oaks: Sage Publ.

KAHIN, B.; KELLER, J. eds. (1997). Coordinating the Internet. Cambridge: MIT Press.

LELONG, B.; MALLARD, A. eds. (2000). La fabrication des normes. Paris: Réseaux, 18: 102.

LESSIG, L. (1999). Code \& Other Laws of Cyberspace. NY: Basic Books.

LOADER, B.D. ed. (1997). The Governance of Cyberspace. London: Routledge.

MACKENZIE, D.; WAJCMAN, J. eds. (1999). The Social Shaping of Technology, 2nd ed.. Buckingham: Open University Press.

MILLERAND, F. (2001). Le courrier électronique: artefact cognitif et dispositif de communication, Colloque La communication médiatisée par ordinateur: un carrefour de problématiques, Congrès de l'ACFAS, U. De Sherbrooke, 15 au 15 mai, texte disponible en ligne: http://grm.uqam.ca/.

NORMAN, D. (1993). Les artefacts cognitifs, Raisons Pratiques, 4, 15-34. 
PROULX, S. (2001). «Usages de l'Internet: la "pensée-réseaux" et l'appropriation d'une culture numérique», in Eric Guichard, éd., Comprendre les usages de l'Internet. Paris: Éditions Rue d'Ulm, ENS, p. 139145 .

(1990). «La promotion sociale de la "culture informatique": du "computer power to the people" à l'ef?cacité d'un nouvel outil pour le travail de bureau», Culture technique, 21, Paris, p. 224-235.

PROULX, S. dir. (1988). Vivre avec l'ordinateur. Les usagers de la micro-informatique, G. Vermette, Montréal.

SNOW, C.P. (1968). Les deux cultures suivi de Supplément aux deux cultures. Paris: Jean-Jacques Pauvert éditeur, [éd.orig. 1959 et 1963].

WELLMAN, B. ed. (1999). Networks in the Global Village. Boulder: Westview Press.

Traduzido por Inês Signorini (UNICAMP)

Recebido: 25/07/2010

Aceito: 27/11/2010 